\title{
Construction of a Survival Prediction Model for High-and Low-Grade Upper Tract Urothelial Carcinoma After Tumor Resection Based on "SEER Database": A Retrospective and Multicenter Study
}

\section{Changgang Sun ( $\sim$ scgdoctor@126.com )}

Qingdao Academy of Chinese Medical Sciences, Shandong University of Traditional Chinese Medicine, Qingdao266112, Shandong, P. R. China; Department of Oncology, Weifang Traditional Chinese Hospital, Weifang261000, Shandong, P. R.China https://orcid.org/0000-0003-0778-8788

\section{Mengmeng Wang}

Clinical Medical Colleges, Weifang Medical University, WeiFang 261000, China

\section{Xin Ren}

Clinical Medical Colleges, Weifang Medical University, WeiFang 261000, China

\section{Ge Wang}

Clinical Medical Colleges, Weifang Medical University, WeiFang 261000, China.

\section{Xiaomin Sun}

Clinical Medical Colleges, Weifang Medical University, WeiFang 261000, China.

\section{Shifeng Tang}

Department of Oncology, Weifang Traditional Chinese Hospital, Weifang 261000, China.

\section{Baogang Zhang}

Department of Pathology, Weifang Medical University, Weifang 261053, China.

\section{Xiaoming Xing}

Department of Pathology, Affiliated Hospital of Qingdao University, Qingdao 266555, China.

\section{Wenfeng Zhang}

Clinical Medical Colleges, Weifang Medical University, WeiFang 261000, China.

\section{Guojun Gao}

Department of Urology, The Affiliated Hospital of Weifang Medical College,WeiFang 261000, China. Jing Du

Department of Urology, Weifang Traditional Chinese Hospital, Weifang 261000, China.

\section{Shukun Zhang}

Department of Pathology,Weihai Municipal Hospital,Cheeloo College of Medicine,Weihai 264200,Shandong University

\section{Lijuan Liu}

Department of Oncology, Weifang Traditional Chinese Hospital, Weifang 261000, China.

\section{Xia Zheng}


Department of Oncology, Weifang Traditional Chinese Hospital, Weifang 261000, China.

\section{Zhenkun Zhang}

Department of Oncology, ShouGuang People's Hospital,Weifang262700,China.

\section{Research}

Keywords: Upper Tract Urothelial Carcinoma, SEER Program, Grade, Nomogram, Tumor Resection, Overall Survival, Multicenter

Posted Date: February 24th, 2021

DOl: https://doi.org/10.21203/rs.3.rs-223524/v1

License: (9) This work is licensed under a Creative Commons Attribution 4.0 International License. Read Full License 


\section{Abstract}

Background: There are difffferences in survival between high-and low-grade Upper Tract Urothelial Carcinoma (UTUC). Our study aimed to develop a nomogram to predict overall survival (OS) of patients with high- and low- grade UTUC after tumor resection, and to explore the diffference between high-and low-grade patients.

Methods: Patients confifirmed to have UTUC between 2004 and 2015 were selected from the Surveillance, Epidemiology and End Results (SEER) database. The UTUCs were identifified and classifified as high- and low-grade, and 1-, 3-and 5-year nomograms were established. The nomogram was then validated using the Chinese multicenter dataset (patients diagnosed in Shandong, China between January 2010 and October 2020).

Findings: In the high-grade UTUC patients, nine important factors related to survival after tumor resection were identifified to construct nomogram. The ability of the model to distinguish between UTUC grades was verifified using two datasets (internal validation dataset, $\mathrm{C}$ index $(95 \% \mathrm{Cl}): 0.729[0.707-0.754]$;Chinese multicenter validation dataset: $\mathrm{C}$ index $(95 \% \mathrm{Cl}): 0.763[0.656-0.869])$. On the other hand, Two independent predictors were identifified to construct nomogram of low-grade UTUC. The $\mathrm{C}$ index was $0.714(95 \% \mathrm{Cl}$ : 0.671-0.758) for the training set,0.731(95\% Cl:0.670-0.791) for the internal validation dataset, and 0.825 (95\% Cl:0.689-1.00) for the Chinese multicenter dataset. Encouragingly, the nomogram was clinically useful and had a good discriminative ability to identify patients at high risk.

Interpretation: We constructed a nomogram and a corresponding risk classifification system predicting the OS of patients with an initial diagnosis of high-and low-grade UTUC.

\section{Full Text}

This preprint is available for download as a PDF.

\section{Figures}




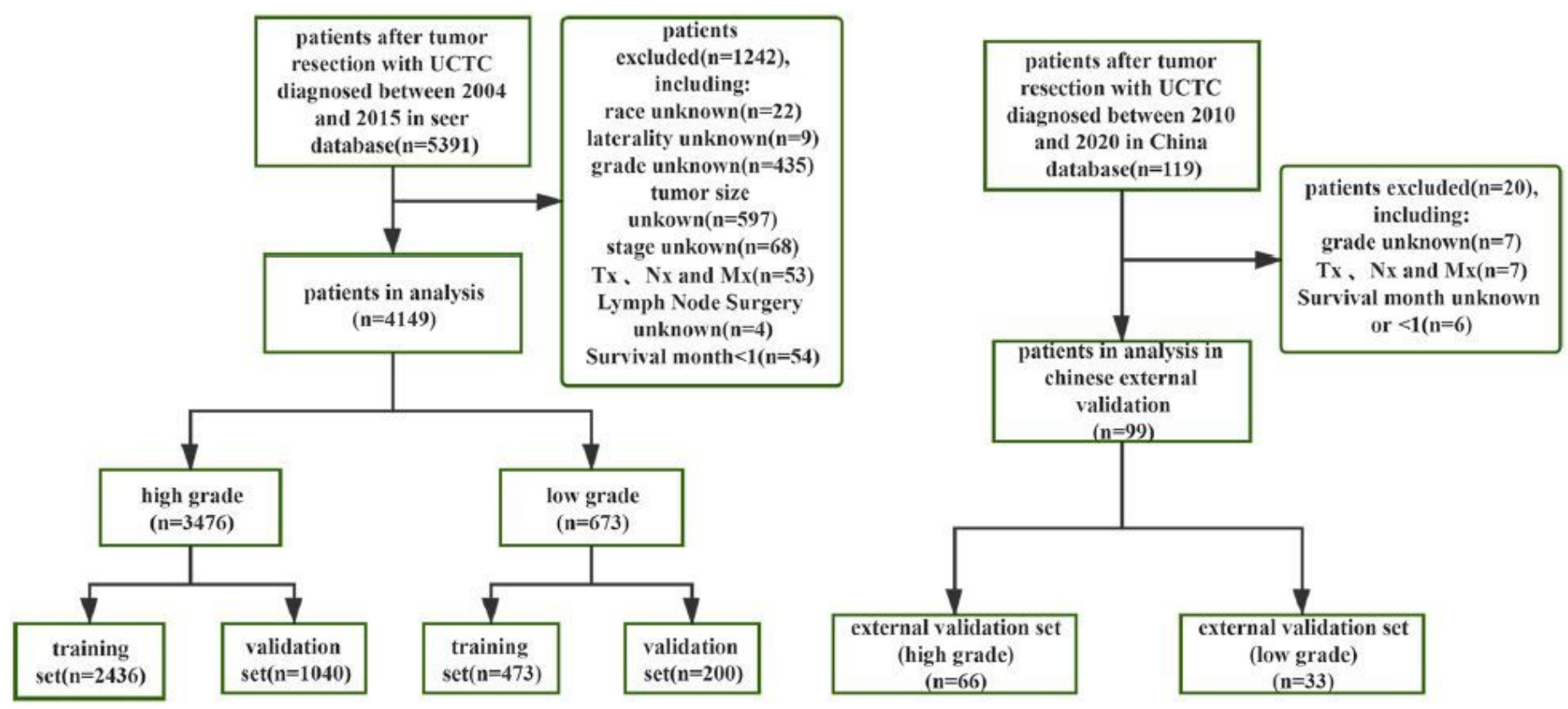

\section{Figure 1}

Flow chart illustrating patient selection for this study. 
(a)

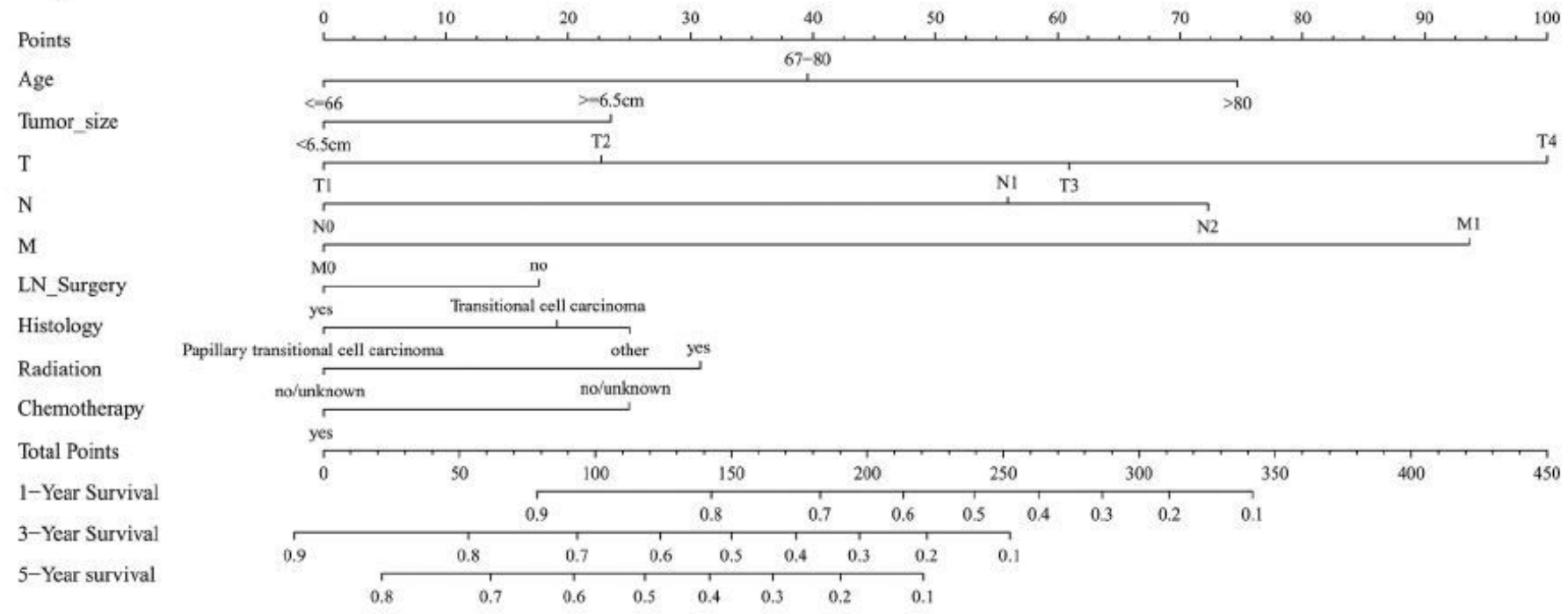

(b)

Points

$\begin{array}{lllll}0 & 10 & 20 & 30 & 40\end{array}$

50

60

70

80

90

100

Age

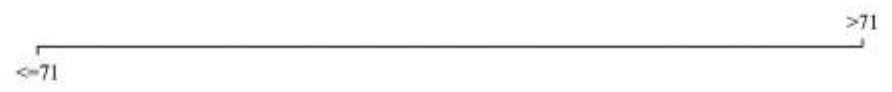

stage

II

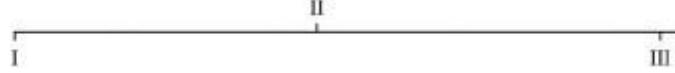

Total Points

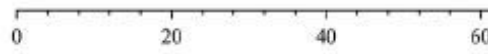

$60 \quad 80$

100

120

140

160

180

1-Year Survival

0.9

3-Year Survival

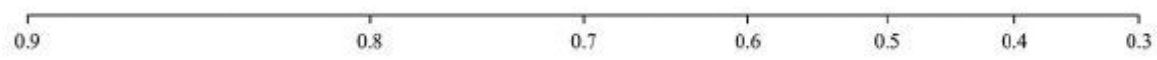

5-Year survival

\begin{tabular}{llllllll}
\hline 0.8 & 0.7 & 1 & 1 & 1 & 1 & 1 & \\
\hline & 0.6 & 0.5 & 0.4 & 0.3 & 0.2 & 0.1
\end{tabular}

\section{Figure 2}

Nomograms to predict the 1-, 3-, and 5-year overall survival of patients with high-grade cancers (a) and low-grade cancers (b). The score for each independent prognostic factor was summed up. Then, the overall survival rate was obtained from the total number of points in the bottom scale for each individual. 

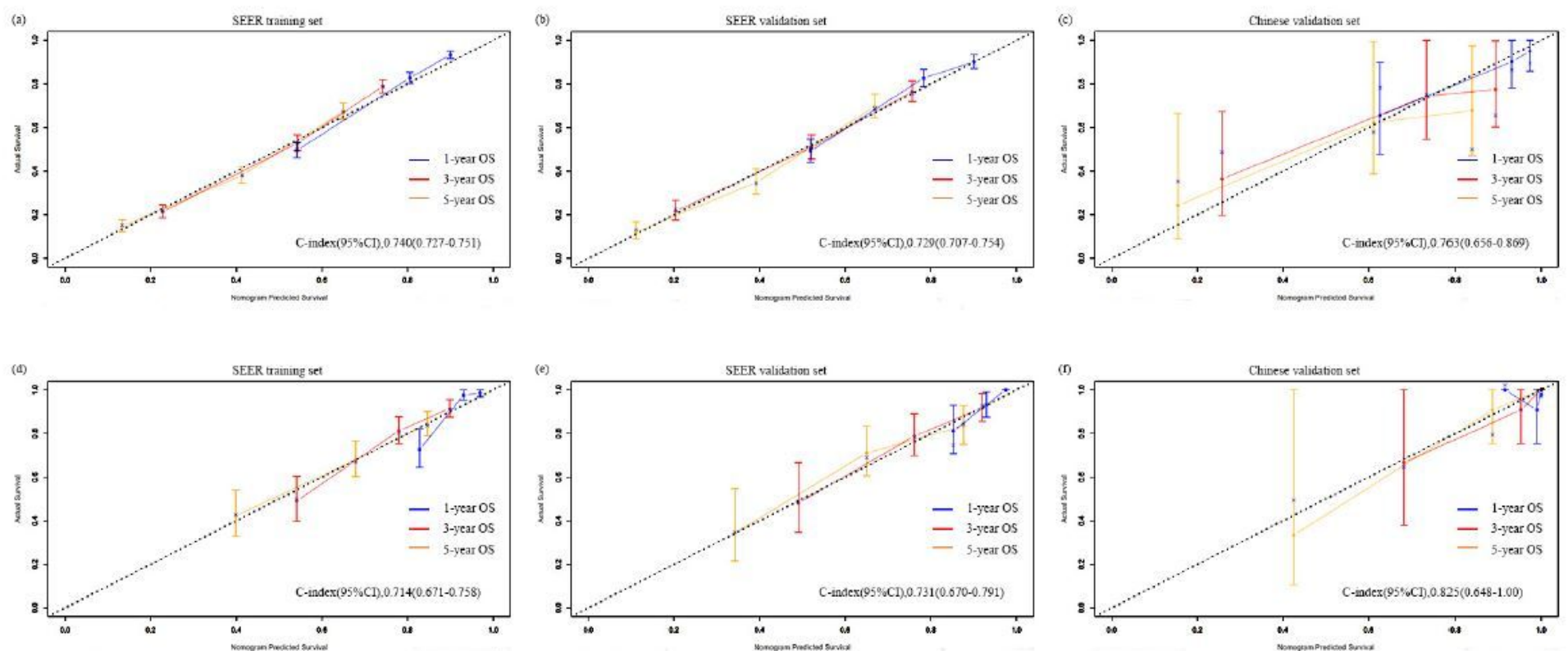

\section{Figure 3}

Calibration plots comparing the similarity between the nomogram-predicted survival rates (represented by $\mathrm{x}$-axis) and the actual survival rates (represented by $\mathrm{y}$-axis).a: 1-, 3-, and 5-year OS for high-grade cancers in the training dataset from the SEER database; b: 1-, 3-, and 5-year OS for high-grade cancers in the validation dataset from the SEER database;c: 1-, 3-, and 5-year OS for high-grade cancers in the validation dataset from the China multicenter dataset;d: 1-, 3-, and 5-year OS for low-grade cancers in the training dataset from the SEER database;e: 1-, 3-, and 5-year OS for high-grade cancers in the validation dataset from the SEER database.f: 1-, 3-, 5-year OS for low-grade cancers in the validation dataset from the China multicenter database. OS: overall survival; SEER: the Surveillance Epidemiology, and End Results database. 

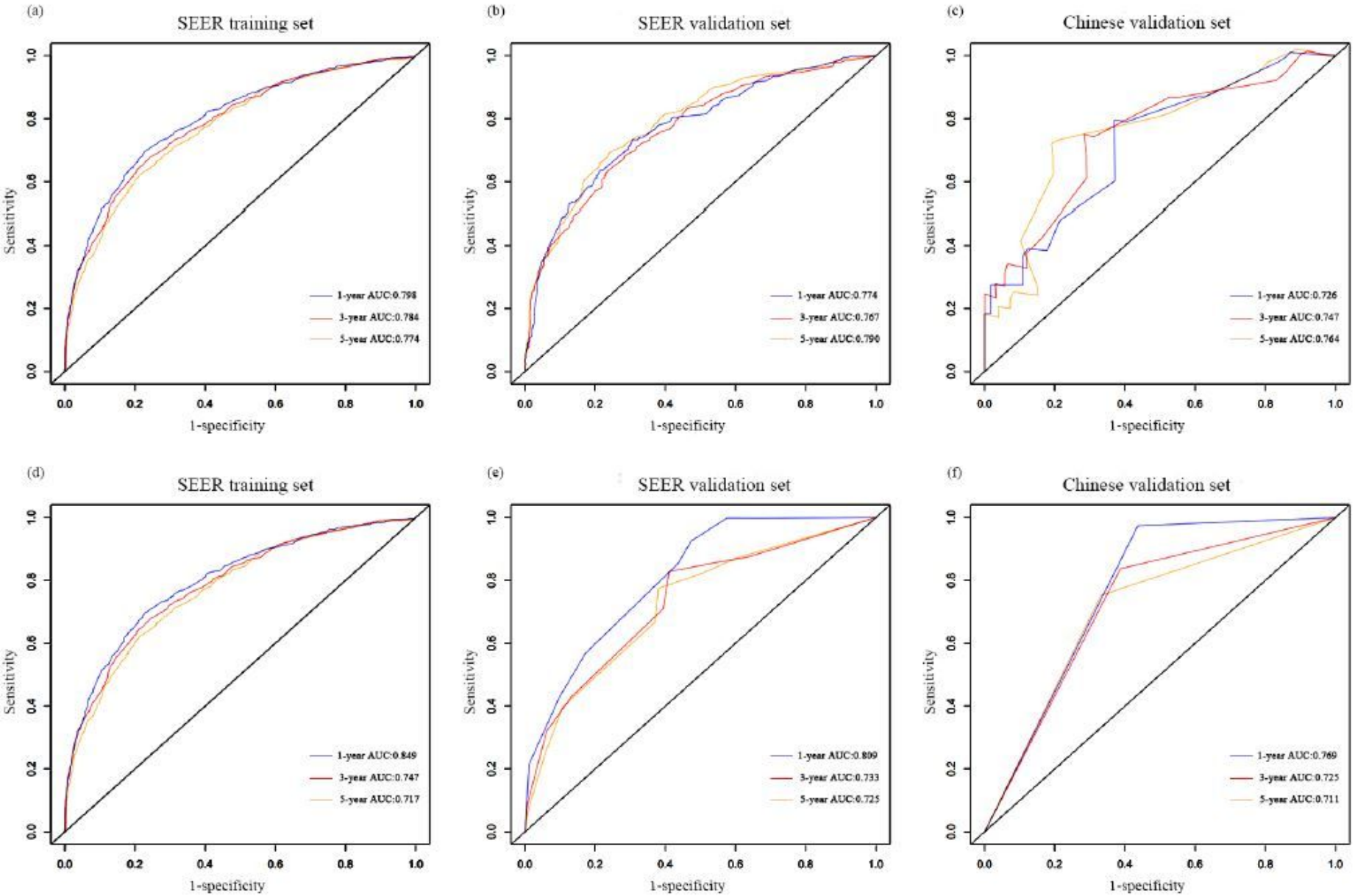

\section{Figure 4}

Comparison of the AUC values of the1-, 3-, and 5-year OS for high-grade and low-grade cancers. The AUC values of the nomograms in the training and validation datasets for OS of high-grade $(a, b)$ and lowgrade (d, e), and the Chinese validation dataset for high-grade (c) and low-grade(f). AUC: area under the curve; OS: overall survival. 
(a)

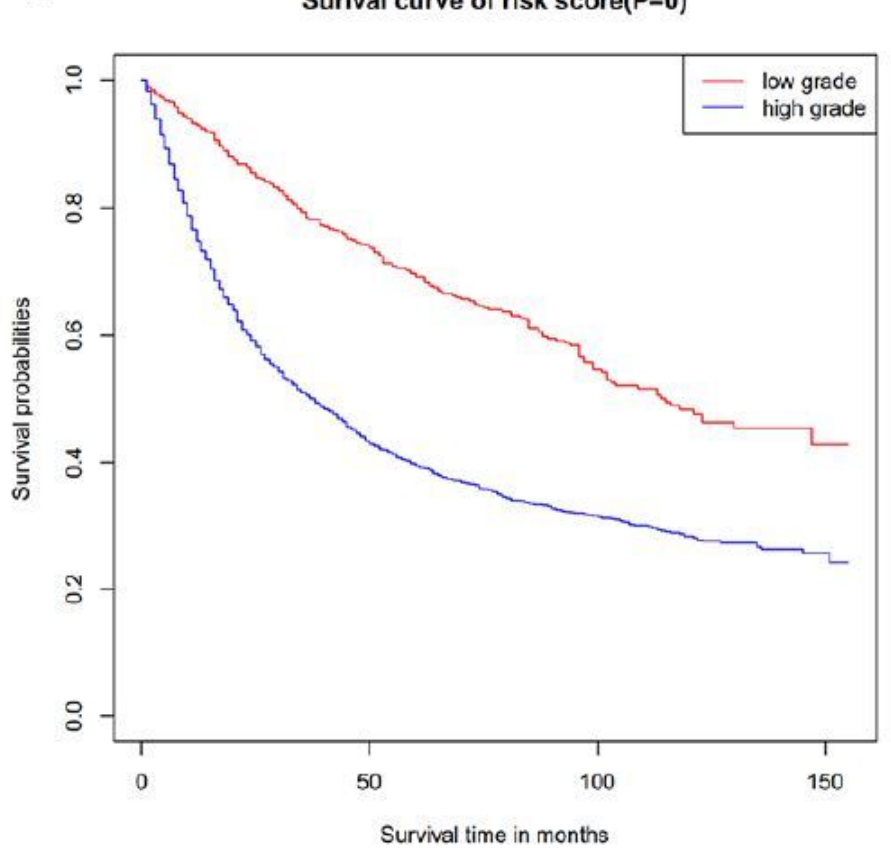

(b)

Surival curve of radation $(P=0)$

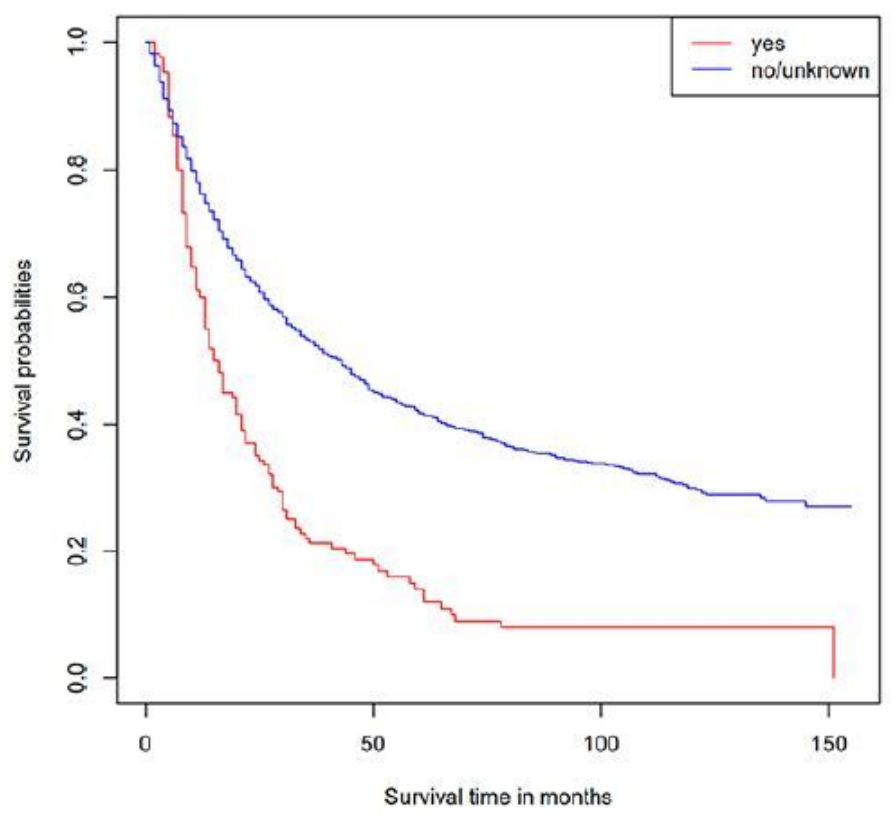

Figure 5

Kaplan-Meier survival curves of patients. Kaplan-Meier survival curves of OS in:(a):high-and low-grade datasets;(b):patients under-going radiotherapy 

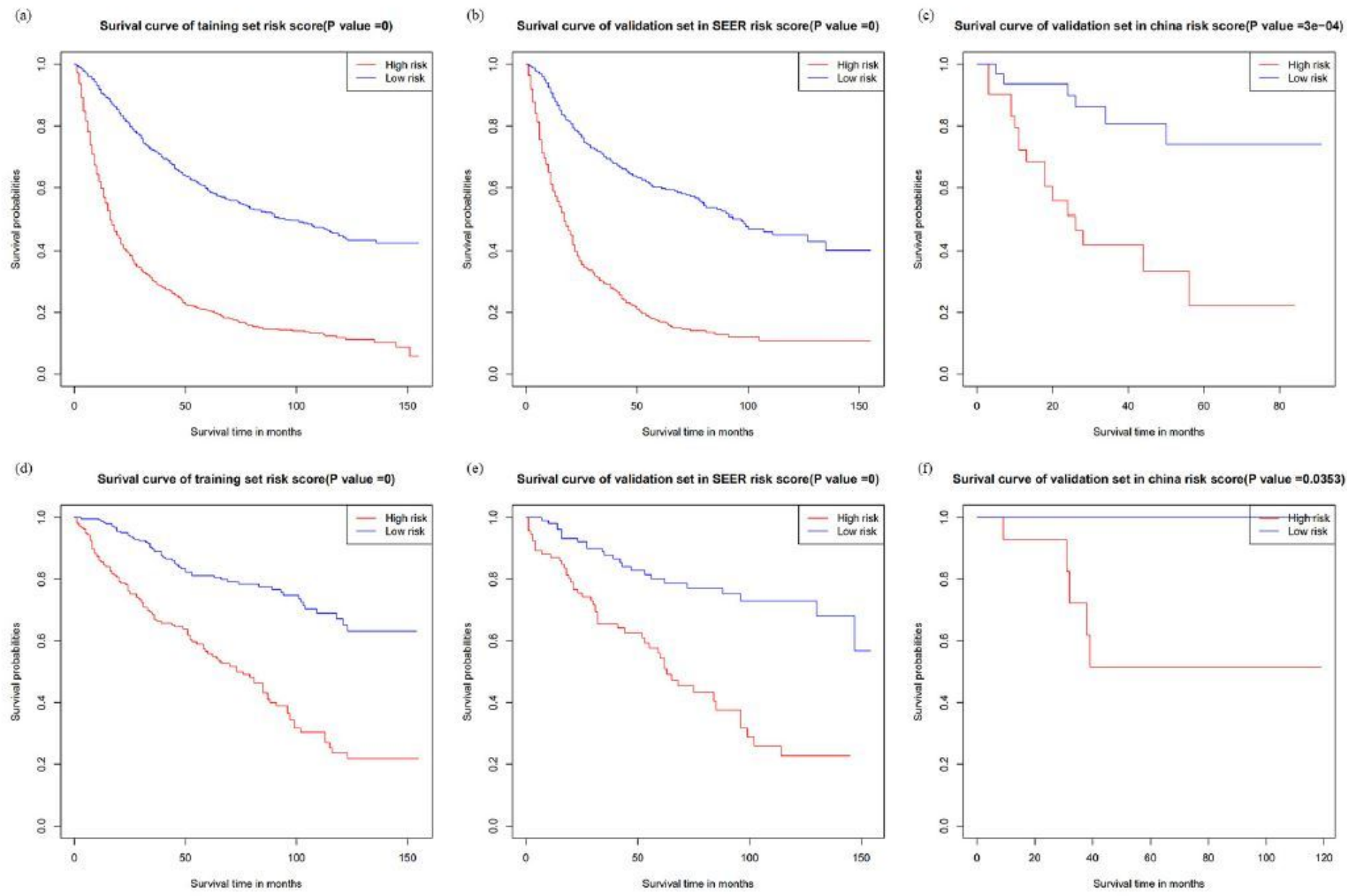

\section{Figure 6}

Kaplan-Meier survival curves categorized into low-risk and high-risk groups. (a):high-grade training dataset;(b):high-grade validation dataset in the SEER database;(c):high-grade validation dataset in the China multicenter database;(d):low-grade training dataset;(e):low-grade validation dataset in the SEER database;(f):low-grade validation set in the Chinese multicenter database. OS: overall survival; SEER: the Surveillance Epidemiology, and End Results database. 


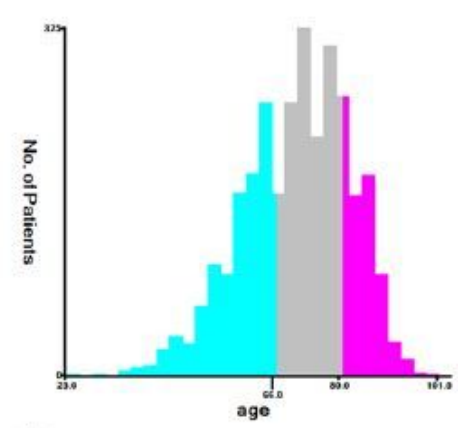

(a)

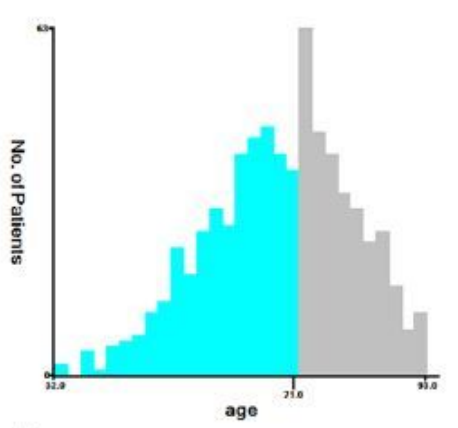

(c)

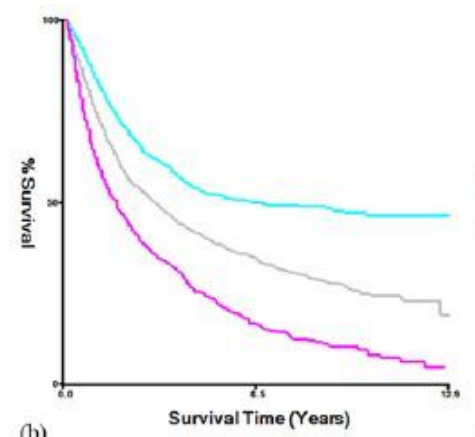

(b)

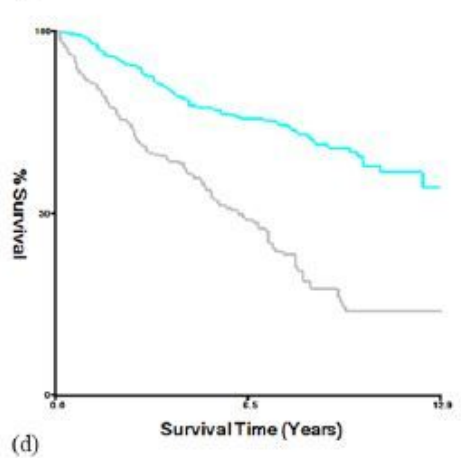

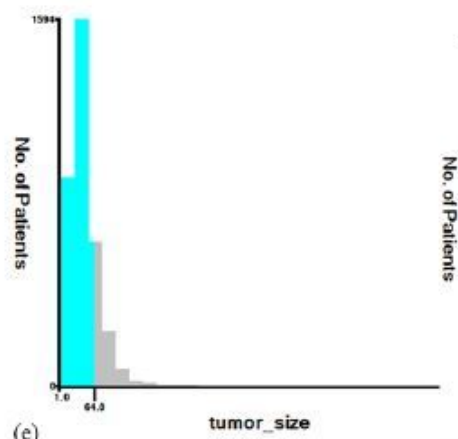

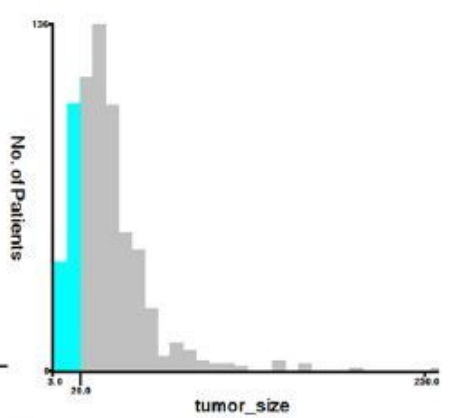

(g)

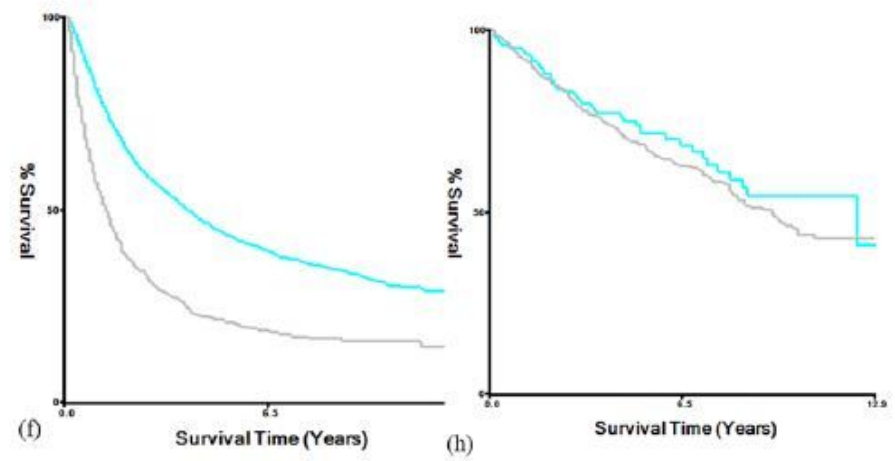

\section{Figure 7}

Appendix Figure (a-d) X-tile plots of age at diagnosis, identifying the best risk score cut-off based on the overall survival (OS) in the high- and low-grades;(e-h)X-tile plots of tumor size, identifying the best risk score cut-off based on the OS in the high- and low-grades. 